\title{
Internet of Things and Online Learning: Intelligent Systems beyond Covid-19
}

\author{
Delali Kwasi Dake \\ University of Education, Winneba \\ P.O. Box 25, UEW, Winneba
}

\author{
Davidson Kwamivi Aidam \\ University of Education, Winneba \\ P.O. Box 25, UEW, Winneba
}

\author{
Verite Ken Agbotse \\ University of Education, Winneba \\ P.O. Box 25, UEW, Winneba
}

\begin{abstract}
The advancements in Internet of Things applications has seen a tremendous growth with 5G and later technologies. The industry 4.0 revolution of digital automation should not exempt education, especially with the ravaging COVID-19 pandemic. The sudden spread of the virus has necessitated a policy direction in online teaching and learning for most academic institutions. The traditional classroom, which has its positives, is minimal in the educational space since distance has become primary in COVID protocols. To wholly integrate traditional classroom merits in online learning, we propose an intelligent online learning system that discovers hidden learner behaviour, and improves personal learning using supervised, unsupervised, and Reinforcement Learning (RL) algorithms. The designed framework automates the online learning space and aids the instructor with lesson planning, delivery approaches, and learner groupings. The learner also constructs knowledge and discovers learning styles through a RL software agent that continuously interacts with the online system using exploration and exploitation mechanisms.
\end{abstract}

\section{General Terms}

Education, Distance Education, Machine Intelligence Systems, COVID-19.

\section{Keywords}

Online Learning; Internet of Things; Covid-19; 5G Networks; Smart Education; Smart Campus; Machine Learning; Big Data

\section{INTRODUCTION}

The deadly COVID-19 pandemic has drastically affected the educational sector, and the fear of the pandemic has hastily transformed academic institutions, especially the online learning space [1][2]. The rapid pandemic wave across the globe forced many institutions to close temporarily, and those without structured online learning platforms paid heavily for their educational lapses and policy directions. According to the World Bank report, COVID-19 has disrupted the studies of over 220 million post-secondary students worldwide, accounting for $13 \%$ of the total number of students affected globally (World Bank, 2020). Even in 2021, most institutions have discontinued the in-person teaching mode and resorted fully to online learning. Other educational institutions struggle to effectively execute the blended mode of teaching and learning with pedagogical considerations [3], especially with the social distancing rule that applies to the pandemic.

Africa has not been exempted from the devasting effects of the pandemic on the economy and education. The eLearning Africa and EdTech Hub (2020) research indicates that 97\% of schools

were closed in Africa during the pandemic, with inadequate appropriate technologies affecting learning. The findings further ascertain the relevance of online educational frameworks in national curriculums to enable effective distance learning in response to unforeseen crises in the future [4]. The effectiveness of online education in Africa needs innovative models with intelligent integration, especially with the endorsed concept of personalised learning [5]. The idea of personalised learning stems from the theory of constructivism, where learners based on pedagogy construct knowledge through active participation in the learning process [6]. The future of online learning needs the integration of emerging technologies, including the advancements in 5G mobile technologies (5G MT), Internet of Things (IoT), and Artificial Intelligence (AI).

The 5G network has reliable use cases that will shift the education system to the online world. Integrating $5 \mathrm{G}$ networks as part of the educational ecosystem will completely revolutionise and enhance the teacher and learner experience. As shown in Fig.1, the 5G deployment in education will provide ultra-reliable and low latency communication, massive machine-type communications, and enhanced mobile broadband [7].

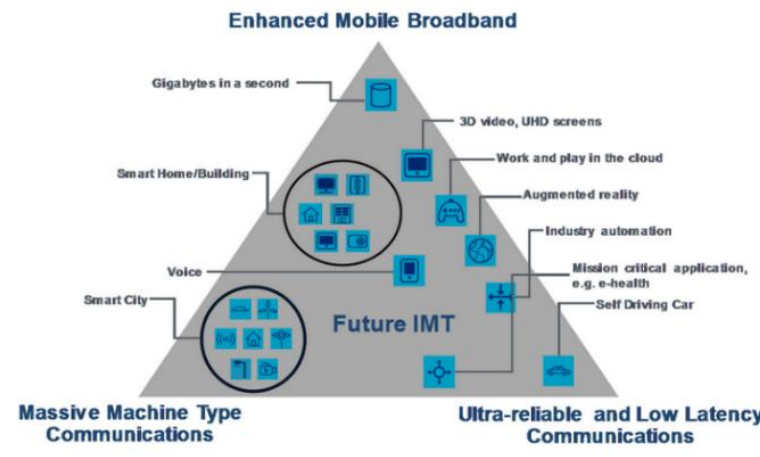

Fig 1: 5G Use Cases

The ultra-reliable and low latency communication (URLLC) will primarily support data transmission in milliseconds and accommodate the emerging services the online learning model will utilise with very low latency [8]. The enhanced mobile broadband (eMBB) will enable higher bandwidth capacity with tens of gigabits per second peak data transfer rates for the end-user of the online learning model [9]. The massive machine type communication (mMTC) will provide connectivity to a larger number of devices the learner and teacher will use to engage the online learning model [10]

The deployment of 5G technologies will speed up the connectivity of billions of objects. According to Ericsson Mobility Report 2021, IoT connections will increase to 26.4 billion in 2026 from the initial estimate of 12.4 billion in 2020 [11]. The compound annual growth rate (CAGR) is $13 \%$ average for Wide-area IoT, Cellular IoT, and Short-range IoT devices. As IoT becomes widespread, educational institutions 
must leverage the technology and implement intelligent systems relevant to stakeholders. Popular IoT implemented technologies from smart buildings to smart healthcare has increased productivity, especially in advanced Countries [12][13], and education should not be an exception. Little research has been done on smart education [14][15][7][16], especially with intelligent online learning systems. The increasing IoT connectivity is estimated to generate 175 zettabytes of data in 2025 from an initial 33 zettabytes in 2018 (Data Age, 2020). This huge data needs machine learning algorithms for pattern identification and prediction. Educational Data Mining (EDM) involves unique data types in educational settings and the application of machine learning algorithms to uncover teaching and learning behaviors [17]. The online learning environment provides a valuable way to extract data from learners and discover hidden patterns [18].

Pattern detection in educational data requires the implementation of machine learning algorithms to automate the process through training models and agents. Machine learning is a branch of AI that involves the ability of machines to learn and evolve without explicit instructions [19]. The supervised machine learning algorithms use labelled datasets to train agents for future predictions [20]. The unsupervised learning methods are not pre-assigned with labels and involve clustering to infer patterns and groupings [21]. Reinforcement Learning (RL) which represents the third class of machine learning algorithms, involves the training of agents through trial and error in an environment to attain mastery with a reward signal [22]. Both supervised and unsupervised algorithms have been used widely in Educational Data Mining (EDM) and achieved good results, especially in predicting students' academic performance, cluster grouping, and student behaviour modelling. Reinforcement Learning implementation in education will significantly impact personalised learning since agents will interact with learners and recommend educational materials through trial and error until mastery. The integration of RL in education has been under-utilised.

In this study, we propose IoT frameworks that will enhance the integration of emerging technologies in online teaching and learning. The research first discussed the relevance of the online learning environment in the face of COVID-19. The second section discussed reviews that are relevant to education. The third section explained the designed models with educational relevance, and the final section recommended actions for policy implementation in educational institutions.

\section{REVIEW OF LITERATURE}

[23] proposed an IoT-based network analysis framework to observe learners' interaction with IoT applications based on the unified theory of acceptance and use of technology 2 (UTAUT2) trust model. The framework structured UTAUT2 incorporation of IoT in Higher Education to include performance expectancy, effort expectancy, social influence, facilitating conditions, hedonic motivation, price value, trust, privacy risk, security risk, and behavioural intention.

[24] designed an intelligent campus framework, iCampus, consisting of the following modules: iSocial, iLearning, iGreen, iHealth, iManagement, iLearning, and iGovernance. The iSocial of the intelligent campus framework involves social networking among learners. The iManagement includes smart building, security surveillance, and emergency response. The iGreen focuses on green ICT, energy conservation, and resource management. The iHealth module monitors learners' health with epidemic alert systems, whiles the iGovernance provides administrative management and change management.

[16] proposed artificial intelligence modules for smart campus. The artificial intelligence powered centralised application (AIPCA) connects admissions in tertiary institutions and is relevant to prospective applicants. The library component, artificial intelligent library assistant smart library (AILA), is a virtual assistant that assists the user in searching for books, publications, and related information. The personal learning companion, artificial intelligence learning companion (AILC), is for classroom learning activities. For AI classroom systems, the Artificial Intelligence in Classroom (AIC) module has an embedded intelligence in all the interactive devices in the classroom.

\section{THE PROPOSED IOT FRAMEWORKS FOR DISTANCE EDUCATION}

The framework adopts emerging technologies including $5 \mathrm{G}$, IoT, and machine learning for an online learning experience that fosters knowledge acquisition and intelligent pattern analytics. The framework is comprehensive to all users of the online learning environment, and its implementation will minimize the traditional classroom approach to teaching and learning.

\subsection{Student Modules (IOT Sensors)}

IoT sensors and devices work at the perception layer of the IoT architecture. The perception layer is the lowest layer of the IoT architecture and is responsible for sensing and gathering information about the environment [25]. Sensors are devices that detect and replace external data with a signal that people and machines can understand. Sensors have made it possible to collect data in virtually any circumstance with low power utilisation.

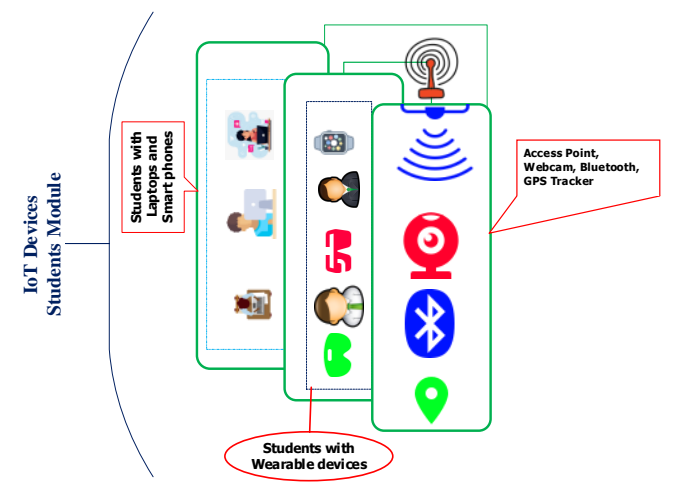

Fig 2: IoT Sensors in Education - Student Module

As shown in Fig. 2, the students' module captures data coming from various IoT devices, including a web camera, a GPS tracker, and a microphone of individual learners. The webcam automatically captures and sends video and image signals to the cloud storage via the IoT Hub whenever a student or an instructor logs into the system. The system keeps track of the activities performed by individual students as well as the lecturers. These activities range from quizzes, exams, lectures as well as live discussions. The signals from the students, including emotional detectors, attention span, and attendance, are sent into the cloud for processing, analysis, and storage. For effective data processing, it is a requirement for students to use computers that support 
webcams before access is granted into the system for an online class activity. The Global Positioning System (GPS) tracker is an IoT sensor embedded in smart devices to monitor the location of students during lessons online. Class attendance has input from the sensors with a boolean representation of being present or absent during lesson delivery. The learner's attention span and emotional strains during lessons or discussions are significant to the success of the learning process. Psychologists claim that the typical student's attention span is about 10 to 15 minutes long [26], yet most university lectures last 2 to 3 hours. It's natural for student attention levels to vary according to motivation, mood, and the perceived relevance of the material [27]. Machine learning and stream analytics within the cloud can detect students' moods and concentration levels based on the video streams from the webcam. This stream of data is being analysed over time as learners continue to interact with the system. The instructor then gets the interpretation of this complex process in the simplest form as shown on the dashboard for decision making and to readjust the teaching methodology if necessary. At equal time intervals, the audio stream from each student's microphone is also examined. Depending on the training activity, appropriate indicators such as voice, noise, pressing keys, are selected in an analogous manner. The wearable gadgets including electroencephalogram (EEG) device, a GPS tracker, a wristwatch and others provides important information to the instructors during lab work, tests, and exams.

\subsection{The Cloud Server - Data Processing, Analysis, and Storage}

The Cloud Server consists of data storage, data analytics, and the environment for Machine Learning, including Reinforcement Learning (RL), as shown in Fig. 3

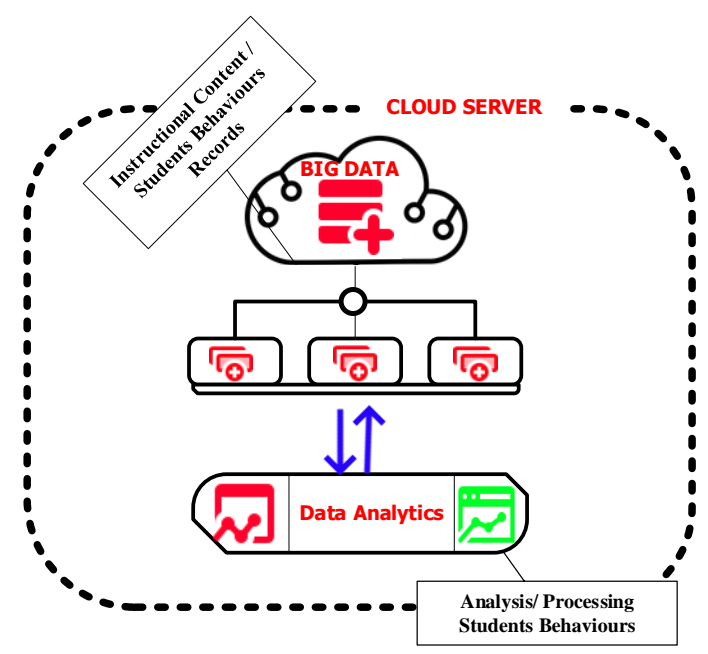

Fig 3: Cloud Server for Data Processing, Analysis, and Storage

The data from learners and the learning contents provided by instructors are stored on the cloud server. The big data engine stores the extreme volume of data from users of the online learning environment with various data types and velocity. The data stored becomes the primary raw data for the data/stream analytics engine and the environment for the RL agents. Data from the sensors via the big data engine are analysed in real-time using the Stream Analytics engine inside the Cloud Server. The primary purpose is to present up-todate information to the instructor and keep the state of data updated. This plays a significant role in checking students' attendance, location of connectivity, facial expression as learners interacts with the system. Stream Analytics ingests incoming data from one or more configured inputs (sensors). Then a query is applied to this data to filter, sort, aggregate, or join other reference data over a while. The archiving stores the raw data to evaluate the environmental conditions and the results sent to the internal stream processor. The results are assessed by triggering the invoke improvement actions. If triggering conditions are satisfied, an improvement action is generated and posted back. All potentially computationally intensive tasks are executed in dedicated containers in a cluster to ensure high performance. For the online study, we recommend the Apache Kafka streaming platform. Apache Spark's big data analytics engine builds evaluations, and the adaption engine is based on docker containers. The data from the stream analytics now serves as the environment for the agents. The data analytics engine also has a clustering algorithm. In the educational sector, internal and external assessments are used to evaluate pupils' academic performance. Class test, lab performance, assignment, quiz, and attendance are examples of internal examinations. The prior semester grade and the final semester grade are external assessments. Using data clustering and classification techniques, the agent can learn and forecast a student's ultimate grade by utilising the learners' internal evaluation and external assessments as the environment. From the literature reviewed, the following data mining algorithms has shown good classification results: the K-means, hierarchical clustering, apriori, decision tree, support vector machines, Naïve Bayes, and the deep learning algorithms [28][29] and forms the data analytics engine.

\subsection{The Reinforcement Learning Agent}

As shown in Fig. 4, the Reinforcement Learning (RL) module forms the basis for personalized learning and recommender system for the online learning framework. The RL agent continuously interacts with the big data and the data analytics engines to learn about the data patterns and generate recommendations for the learner and the instructor through a trial and error approach. Based on the feedback from the environment, the agent recommends another set of actions (learning materials) for the users. At each state of the environment, the RL agent suggests different learning methods to the learner and awaits performance feedback. If the feedback is positive, the agent continuously recommends relevant materials to the learner based on the learning method in an exploration-exploitation tradeoff [29]. The RL agent outlines the best behavior models for different scenarios relying on a reward that teaches the agent to choose the most appropriate actions. Per the proposed framework, the agent encounters punishments when the recommended learning material gives a less favourable assessment performance results.

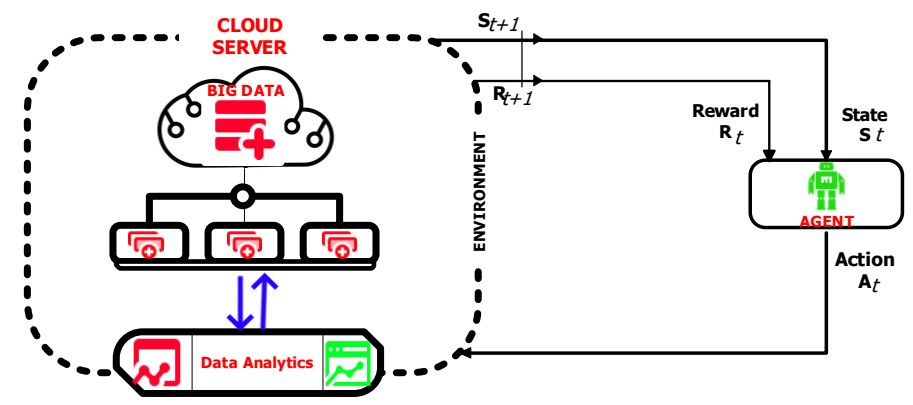

Fig 4: Reinforcement Learning Module 
Thus, the agent learns to recommended learning materials that are appropriate for each learner over time. The integration of RL agents forms the basis of learning automation with personalized learning and constructivism. Incorporating Reinforcement Learning into the teaching and learning process will guide learners and the instructor. The agent provides many learning materials in digital format (texts, videos, tests, podcasts) to build the action recommender system. Feedback is generated to the lecturer to adjust teaching patterns and adopt different learning styles during lesson delivery. The agent is expected to analyze the interests of online students, make predictions about their further needs, and give recommendations on courses. Learning existing experience and the accurate level of knowledge will be reflected correctly by the agent. The agent measures each student's progress, corrects the learning plan and invents the methodology to suit individual learners. It then makes necessary predictions to the instructor on actions to take to improve learning participation and performance.The complete framework in Fig. 5 includes the instructor module, the learner module, and the machine learning engine that have been discussed in the previous section.

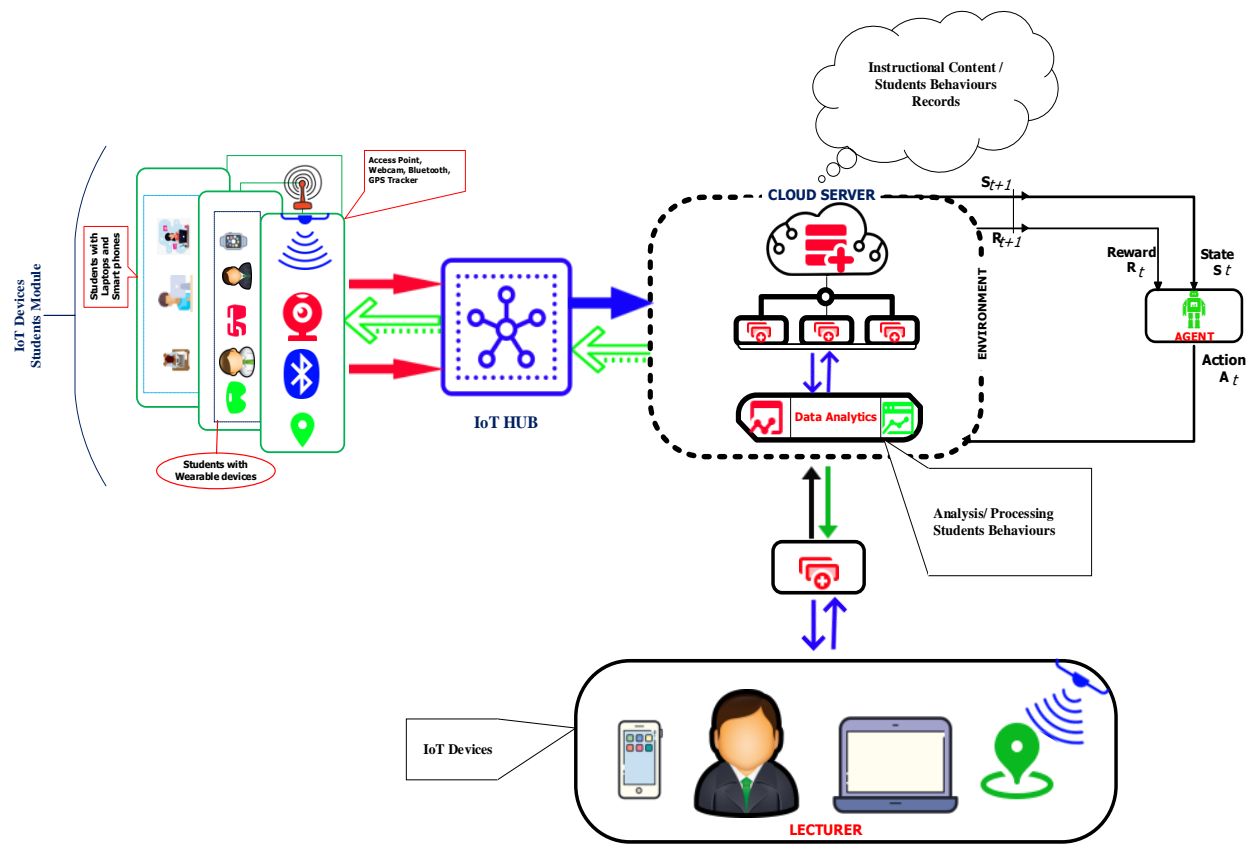

Fig 5: Proposed IoT framework for Online or Distance Learning

\section{CONCLUSION}

The negative impact of the pandemic has necessitated many proposed solutions in the online learning space for educational institutions. Most institutions have resorted fully or partially to the virtual mode of lesson delivery across the globe. Some institutions have also strengthened its distance educational model to improve learner and instructor activity modules online. The rapid emergence of Internet of Things and related technologies, when implemented, will improve the online learning environment and provide intelligence for the instructor about individual learners and their learning patterns. Instructor lesson preparation and reflective teaching philosophy will be adapted to suit each learner and, over time, become a big data hub for pattern discovery using machine learning algorithms. We developed a framework that ascertains the relevance of machine learning, IoT, RL, and big data in education. The framework has a module for data generation mechanisms from the IoT devices integrated within the learner and instructor machines. The big data generated is relevant in tracking patterns using machine learning algorithms to discover unknown patterns about learners and instructors. For personalized learning and constructivism, we developed a RL software agent module that progressively uses rewards and punishment to affect each learner state.

\section{REFERENCES}

[1] S. Dhawan, "Online Learning: A Panacea in the Time of COVID-19 Crisis," J. Educ. Technol. Syst., vol. 49, no. 1, pp. 5-22, 2020, doi: 10.1177/0047239520934018.
[2] D. Nambiar, "The impact of online learning during COVID-19: students' and teachers' perspective," Int. J. Indian Psychol., vol. 8, no. 2, pp. 783-793, 2020, doi: 10.25215/0802.094.

[3] W. I. O'Byrne and K. E. Pytash, "Hybrid and Blended Learning: Modifying Pedagogy Across Path, Pace, Time, and Place," J. Adolesc. Adult Lit., vol. 59, no. 2, pp. 137140, 2015, doi: 10.1002/jaal.463.

[4] D. K. Dake, D. D. Essel, and J. E. Agbodaze, "Using Machine Learning to Predict Students' Academic Performance During Covid-19," no. Dm, pp. 9-15, 2021, doi: 10.1109/iccma53594.2021.00010.

[5] V. Prain et al., "Personalised learning: Lessons to be learnt," Br. Educ. Res. J., vol. 39, no. 4, pp. 654-676, 2013, doi: 10.1080/01411926.2012.669747.

[6] J. G. Elliott, N. Hufton, L. Illushin, and F. Lauchlan, "Motivation in the Junior Years: international perspectives on children's attitudes, expectations and behaviour and their relationship to educational achievement," Oxford Rev. Educ., vol. 27, no. 1, pp. 37 68, 2001, doi: 10.1080/3054980020030583.

[7] D. K. Dake and B. A. Ofosu, "5G enabled technologies for smart education," Int. J. Adv. Comput. Sci. Appl., vol. 10, no. 12, pp. 201-206, 2019, doi: 10.14569/ijacsa.2019.0101228.

[8] N. Javaid, A. Sher, H. Nasir, and N. Guizani, "Intelligence in IoT-Based 5G Networks: Opportunities 
and Challenges," IEEE Commun. Mag., vol. 56, no. 10, pp. 94-100, 2018, doi: 10.1109/MCOM.2018.1800036.

[9] H. Gamage, N. Rajatheva, and M. Latva-Aho, "Channel coding for enhanced mobile broadband communication in 5G systems," EuCNC 2017 - Eur. Conf. Networks Commun., pp. 1-6, 2017, doi: 10.1109/EuCNC.2017.7980697.

[10] I. Jovović, I. Forenbacher, and M. Periša, "Massive Machine-Type Communications: An Overview and Perspectives Towards 5G," Proc. 3rd Int. Virtual Res. Conf. Tech. Discip., vol. 3, no. Ic, pp. 32-37, 2015, doi: 10.18638/rcitd.2015.3.1.73

[11] D. K. Dake, J. D. Gadze, and G. S. Klogo, "DDoS and Flash Event Detection in Higher Bandwidth SDN-IoT using Multiagent Reinforcement Learning," pp. 16-20, 2021, doi: 10.1109/iccma53594.2021.00011.

[12] S. B. Baker, W. E. I. Xiang, S. Member, and I. A. N. Atkinson, "Internet of Things for Smart Healthcare.pdf," IEEE Access, vol. 5, pp. 26521-26544, 2017.

[13] X. Li, R. Lu, X. Liang, X. Shen, J. Chen, and X. Lin, "Smart community: An internet of things application," IEEE Commun. Mag., vol. 49, no. 11, pp. 68-75, 2011, doi: 10.1109/MCOM.2011.6069711.

[14] H. Singh and S. J. Miah, "Smart education literature: A theoretical analysis," Educ. Inf. Technol., vol. 25, no. 4, pp. 3299-3328, 2020, doi: 10.1007/s10639-020-10116-4.

[15] Z. T. Zhu, M. H. Yu, and P. Riezebos, "A research framework of smart education," Smart Learn. Environ., vol. 3, no. 1, 2016, doi: 10.1186/s40561-016-0026-2.

[16] D. Kwasi and A. Halil, "Artificial Intelligence Modules for Higher Educational Institutions," Int. J. Comput. Appl., vol. 178, no. 34, pp. 17-21, 2019, doi: 10.5120/ijca2019919205.

[17] C. Romero and S. Ventura, "Educational data mining: A review of the state of the art," IEEE Trans. Syst. Man Cybern. Part C Appl. Rev., vol. 40, no. 6, pp. 601-618, 2010, doi: 10.1109/TSMCC.2010.2053532.

[18] S. K. Mohamad and Z. Tasir, "Educational Data Mining: A Review," Procedia - Soc. Behav. Sci., vol. 97, pp. 320-324, 2013, doi: 10.1016/j.sbspro.2013.10.240.

[19] S. Chang, T. Cohen, and B. Ostdiek, "What is the machine learning?," Phys. Rev. D, vol. 97, no. 5, p.
56009, 2018, doi: 10.1103/PhysRevD.97.056009.

[20] O. F.Y, A. J.E.T, A. O, H. J. O, O. O, and A. J, "Supervised Machine Learning Algorithms: Classification and Comparison," Int. J. Comput. Trends Technol., vol. 48, no. 3, pp. 128-138, 2017, doi: 10.14445/22312803/ijctt-v48p126.

[21] K. Hsu, S. Levine, and C. Finn, "Unsupervised learning via meta-learning," 7th Int. Conf. Learn. Represent. ICLR 2019, 2019.

[22] D. K. Dake, J. D. Gadze, G. S. Klogo, and H. Nunoomensah, "Multi-Agent Reinforcement Learning Framework in SDN-IoT for Transient Load Detection and Prevention," 2021.

[23] H. Shaikh, M. S. Khan, Z. A. Mahar, M. Anwar, A. Raza, and A. Shah, "A conceptual framework for determining acceptance of internet of things (IoT) in higher education institutions of Pakistan," 2019 Int. Conf. Inf. Sci. Commun. Technol. ICISCT 2019, pp. 7 11, 2019, doi: 10.1109/CISCT.2019.8777431.

[24] B. Hirsch and J. W. P. Ng, "Education beyond the cloud: Anytime-anywhere learning in a smart campus environment," 2011 Int. Conf. Internet Technol. Secur. Trans. ICITST 2011, no. October, pp. 718-723, 2011.

[25] C. Le Zhong, Z. Zhu, and R. G. Huang, "Study on the IOT Architecture and Access Technology," Proc. - 2017 16th Int. Symp. Distrib. Comput. Appl. to Business, Eng. Sci. DCABES 2017, vol. 2018-Septe, pp. 113-116, 2017, doi: 10.1109/DCABES.2017.32.

[26] K. Wilson and J. H. Korn, "Attention during Lectures: Beyond Ten Minutes," Teach. Psychol., vol. 34, no. 2, pp. 85-89, 2007, doi: 10.1080/00986280701291291.

[27] W. Xu, "Learning styles and their implications in learning and teaching," Theory Pract. Lang. Stud., vol. 1, no. 4, pp. 413-416, 2011, doi: 10.4304/tpls.1.4.413-416.

[28] A. Dutt, "Clustering Algorithms Applied in Educational Data Mining," Int. J. Inf. Electron. Eng., no. March, 2015, doi: 10.7763/ijiee.2015.v5.513.

[29] I. J. Sledge and J. C. Principe, "Balancing exploration and exploitation in reinforcement learning using a value of information criterion," ICASSP, IEEE Int. Conf. Acoust. Speech Signal Process. - Proc., pp. 2816-2820, 2017, doi: 10.1109/ICASSP.2017.7952670. 